\title{
The soluble terminal complement complex (SC5b-9) up-regulates osteoprotegerin expression and release by endothelial cells: implications in rheumatoid arthritis
}

\author{
Federica Corallini ${ }^{1, *}$, Fleur Bossi ${ }^{1, *}$, Arianna Gonelli ${ }^{2}$, Claudio Tripodo ${ }^{3}$, Gabriella Castellino ${ }^{4}$, \\ Tom E. Mollnes ${ }^{5}$, Francesco Tedesco ${ }^{1}$, Lucia Rizzi ${ }^{1}$, Francesco Trotta ${ }^{4}$, Giorgio Zauli ${ }^{2}$ and \\ Paola Secchiero ${ }^{2}$
}

\begin{abstract}
Objective. Complement activation products contribute to a large number of inflammatory diseases, including RA. We have investigated whether osteoprotegerin (OPG) may concur with the soluble terminal complement complex (SC5b-9) to the inflammatory cascade characterizing RA.

Methods. Levels of SC5b-9 and OPG in the plasma and SF of patients with active RA were determined by ELISA. The presence of SC5b-9 and OPG in RA synovial lesions was analysed by immunohistochemistry. Cultured endothelial cells were used for in vitro leucocyte/ endothelial cell adhesion assays. In addition, endothelial cells were exposed to SC5b-9 in order to evaluate the effects on the production of OPG protein, as well as the activation of the OPG promoter.

Results. Patients affected by active RA are characterized by elevated levels of both SC5b-9 and OPG in plasma and/or SF. Of note, we have observed a co-localization of SC5b-9 and OPG in endothelial cells of post-capillary venules of RA synovial lesions. Data on endothelial cell cultures showed that exposure to SC5b-9 induced the up-regulation of OPG expression/release, stimulating the transcriptional activity of the OPG promoter, and synergized with TNF- $\alpha$ in up-regulating OPG production.

Conclusions. Our findings demonstrate that SC5b-9 induces OPG production by endothelial cells and we propose that the SC5b-9-mediated up-regulation of OPG may be an important mechanism whereby complement contributes in promoting and/or enhancing the inflammation in RA.
\end{abstract}

KeY wORDS: Endothelium, Osteoprotegerin, Inflammation, Complement system.

\section{Introduction}

The importance of complement system in the development and amplification of the inflammatory process at the tissue level in various pathological conditions, including RA, has been previously demonstrated [1-3]. The pathogenesis of RA is a multi-step event and, besides the contribution of both cell-mediated and antibody-dependent tissue damage, complement has been recognized as one of the key initial events occurring in the acute phase of RA and it may also be responsible for amplification of the inflammatory process occurring during progression of RA [1-3]. Complement activation products are detected both in the circulation [4-6] and in the SF of patients with RA, and their contribution to damage of the synovial membrane is supported by detection of complement deposits in synovial tissue during the chronic phase of RA [7-10]. Moreover, the beneficial effect of anti-complement therapy has been demonstrated in animal models of arthritis [11-13], which share with human RA the features of an inflammatory synovitis characterized by synovial hyperplasia and leucocyte infiltration and, as in the chronic phase of RA, pannus formation and cartilage/bone erosion. In particular, increasing evidence from our group show that the cytolytically inactive soluble terminal complement complex (SC5b-9) exerts

${ }^{1}$ Interdepartmental Center of Molecular Medicine, University of Trieste, Trieste, ${ }^{2}$ Department of Morphology and Embryology, University of Ferrara, Ferrara, ${ }^{3}$ Department of Human Pathology, University of Palermo, Palermo, ${ }^{4}$ Rheumatology Section, Department of Clinical and Experimental Medicine, University of Ferrara, Ferrara, Italy and ${ }^{5}$ Institute of Immunology, Rikshospitalet University Hospital, Oslo, Norway.

Submitted 7 October 2008; revised version accepted 5 December 2008

*Federica Corallini and Fleur Bossi equally contributed to this work.

Correspondence to: Paola Secchiero, Department of Morphology and Embryology, University of Ferrara, Via Fossato di Mortara 66, 44100 Ferrara, Italy. E-mail: paola.secchiero@ unife.it several pro-inflammatory responses acting directly on endothelium, such as the induction of adhesion molecules [14] and the increase of vascular leakage [15].

Osteoprotegerin (OPG) is a soluble member of the TNF receptor super-family that has been originally characterized for its ability to suppress osteoclast formation [16] by binding to RANK ligand (RANKL), and preventing the interaction of RANKL with its high-affinity transmembrane receptor, namely RANK [17]. OPG also interacts with TNF-related apoptosis inducing ligand (TRAIL) [18], a death-inducing ligand whose extracellular domain shares $\sim 30 \%$ homology with RANKL and shows the ability to interfere with the osteoclast formation induced by RANKL [19]. Several reports have previously shown increased serum levels of OPG, as well as of RANKL, in patients affected by RA [20-26], but the physiopathological significance of the up-regulation of OPG in RA patients is not well understood. Interestingly, a possible pathogenetic link between elevated levels of OPG and inflammation has been suggested by recent in vitro studies of our group [27] and Mangan et al. [28] demonstrating that recombinant OPG promotes leucocyte adhesion to endothelial cells.

On this basis, the aim of our study was to investigate potential link between SC5b-9 and OPG in the promotion of the inflammatory cascade characterizing RA, by evaluating the possibility that SC5b-9 might modulate the expression and/or release of OPG in endothelial cells.

\section{Materials and methods}

\section{Patients' samples}

Plasma and SF were obtained from 10 patients with active RA (44-72 years, three males/seven females) fulfilling the ACR classification criteria [29]. The mean disease activity score was 3.2 and the mean disease duration was 4.5 years. All patients were treated with systemic corticosteroids and, in addition, with leflunomide, or HCQ, or rituximab, or MTX. From each patient, plasma was 
obtained from venous blood, while SF was obtained during joint aspiration from knee joints, and collected in heparinized syringes. Venous blood and SF samples were centrifuged at 370 and $250 \mathrm{~g}$, respectively, for $10 \mathrm{~min}$ and the resulting supernatant was stored at $-80^{\circ} \mathrm{C}$, in multiple aliquots, until analysis. As control, plasma was collected from 20 age- and sex-matched healthy donors. Written consent for all procedures was obtained from all subjects in accordance with the Declaration of Helsinki and the institutional review board of the University Hospital of Ferrara gave ethical approval for the study.

\section{ELISA}

OPG levels were measured in plasma and SF samples as well as in endothelial cell culture supernatants using a sandwich-type ELISA kit purchased from Alexis Biochemicals (Lausen, Switzerland), as previously described [30]. The presence and the levels of SC5b-9 into the plasma and SF were evaluated by ELISA as previously described [14], while the levels of TNF- $\alpha$ were analysed by using an ELISA kit purchased from R\&D Systems (Minneapolis, MN, USA). All measurements were done in duplicate and the results were read at an optical density of $450 \mathrm{~nm}$ using an Anthos 2010 ELISA reader (Anthos Labtec Instruments Ges.m.b.H, Salzburg, Austria).

\section{Immunohistochemistry detection of SC5b-9 and OPG}

Samples of synovial membranes $(n=5)$ were gathered from the archives of the Department of Human Pathology of the University of Palermo, as approved by the local institutional review board. All specimens were fixed for $24 \mathrm{~h}$ with $10 \%$ buffered formalin, paraffin-embedded and cut in serial sections of $5 \mu \mathrm{m}$. Tissue morphology was evaluated by haematoxylin and eosin staining. Immunohistochemical detection of SC5b-9 and OPG was performed on serial sections by using the neoepitope-specific anti-C9 antibody (clone WU 13-15; kind gift of Prof. R. Wuerzner, Austria), and the anti-human OPG monoclonal antibody (clone 69 146; R\&D Systems), respectively. After dewaxing and endogenous peroxidase inhibition, slides were microwave-oven heated three times for $5 \mathrm{~min}$ in TRIS/EDTA pH 9.0 buffer (heat-induced epitope retrieval) and then washed with phosphate-buffered saline (PBS). After $30 \mathrm{~min}$ incubation with the primary antibodies (diluted in PBS) at $37^{\circ} \mathrm{C}$, followed by at least two PBS washings, the Novolink Polymer HRP IHC detection kit (Novocastra, UK) was used for $30 \mathrm{~min}$ at room temperature, according to the manufacturer's instructions. Romulin-aminoethylcarbazole was used as a chromogen for $5 \mathrm{~min}$ at room temperature with subsequent nuclear counterstaining with haematoxylin. Negative controls (omission of the primary antibody, and use of an irrelevant antibody of the same isotype as the primary antibody) were included in each immunohistochemical labelling run.

\section{Cell cultures and treatments}

Human umbilical vein endothelial cells (HUVECs) were purchased from BioWhittaker (Walkersville, MD, USA) and grown on $0.2 \%$ gelatin-coated tissue culture plates in M199 endothelial growth medium supplemented with $20 \%$ fetal bovine serum, $10 \mu \mathrm{g} / \mathrm{ml}$ heparin and $50 \mu \mathrm{g} / \mathrm{ml}$ ECGF (all from BioWhittaker). In all experiments, cells were used between the $3 \mathrm{rd}$ and 5 th passage in vitro, as previously described [31]. EDTA-blood samples for the isolation of primary polymorphonuclear neutrophils (PMNs) were drawn from healthy volunteers after obtaining informed consent. Blood samples were diluted 1:2 with PBS and a first separation step was performed by Histopaque ficoll gradient centrifugation (Lymphoprep; Nycomed, Oslo, Norway). PMNs in the pellet of the gradient were separated from erythrocytes by further steps consisting of dextran sedimentation and hypotonic lysis, resulting in purity $>90 \% \mathrm{CD} 15 / \mathrm{CD}_{1} 1 \mathrm{~b}^{+}$cells, as assessed by flow cytometry using specific FITC- or phicoeritrin (PE)-conjugated monoclonal antibodies (Immunotech, Marseille, France; Miltenyi Biotech GmbH, Bergish Gladbach, Germany).

For cell treatments, the following reagents have been used: recombinant human OPG and TNF- $\alpha$ (both purchased from R\&D Systems), and SC5b-9, prepared and purified as previously described [15].

\section{Endothelial-leucocyte adhesion assay}

Vascular endothelial cells were grown to confluence in 24- or 96-well tissue culture plates and stimulated for $16 \mathrm{~h}$ at $37^{\circ} \mathrm{C}$ with SC5b-9 or recombinant OPG. After three washings with serumfree medium, untreated $1-3.5 \times 10^{5} \mathrm{PMN}$ cell suspensions were added to each well and were further incubated at $37^{\circ} \mathrm{C}$ for $30 \mathrm{~min}$. After endothelial-leucocyte co-culture, non-adherent PMNs were removed by washing the wells at least twice. Endothelialleucocyte co-cultures were photographed under a light microscope (10× magnification). The number of adhered PMNs was evaluated by a colorimetric assay using tetramethyl benzidine (Sigma Chemicals, St Louis, CA, USA) as a substrate for myeloperoxidase, as previously described [32], and/or by scoring at least six random fields for each treatment. In the assays, the viability of both endothelial cells and adherent PMNs was routinely monitored at light microscopy by Trypan blue dye exclusion or by flow cytometry after propidium iodide (PI) staining, performed as previously described [33, 34].

\section{Transfection experiments}

To generate the OPG-promoter reporter plasmid (pOPG-Luc) a 1172 -bp fragment of the OPG gene 5 flanking region was amplified from HUVEC genomic DNA by PCR using the following primers $5^{\prime}$-AGATCTCTGGAGACATATAACTTGAAC ACTTGGCCC-3' and $5^{\prime}$-GAATTCTGTGGTCCCCGGAAACC TCAGG-3' (restriction sites are underlined). This fragment was cloned into $B g / \mathrm{II} / E c o$ RI sites in the pMetLucReporter Vector (Clontech, Mountain View, CA, USA). Subconfluent HUVECs were detached and resuspended in the specified electroporation buffer to a final concentration of $7 \times 10^{5}$ cells $/ \mathrm{ml}$. Two micrograms of luciferase-reporter plasmids (either pOPG-Luc or control vector pMetLuc-Reporter, Clontech) were mixed with $0.1 \mathrm{ml}$ of cell suspension, transferred to electroporation cuvettes and nucleofected with the human HUVEC nucleofector kit (Amaxa, Cologne, Germany) using the program A-034 of the nucleofector device (Amaxa NucleofectorII apparatus). In parallel, cells were transfected using the control EGFP-plasmid (Amaxa) and transfection efficiency was monitored in each experiment by scoring the percentage of fluorescent EGFP-positive cells. After electroporation, cells were immediately transferred to complete M199 medium, cultured in 24-well plates and allowed to recover before treatment with SC5b-9. The secreted luciferase was measured with the ready-to-glow secreted luciferase reporter system (Clontech) according to the manufacturer's instructions at $405 \mathrm{~nm}$ in a luminometer, and as previously described [35]. Results were normalized for transfection efficiency, determined in each experiment by co-transfection of a $\beta$-galactosidase-control plasmid under the control of a constitutive promoter (SV40) ( $\beta$-gal Reporter System, Clontech).

\section{Assay for $N F-\kappa B$ DNA binding}

$\mathrm{NF}-\kappa \mathrm{B}$ induction was measured using the Trans-AM NF- $\kappa \mathrm{B}$ p65 kit (Active Motif, Rixensart, Belgium), which measures the level of active form of NF- $\kappa \mathrm{B}$ contained in cell extracts, able to specifically bind to an oligonucleotide containing the NF- $\kappa \mathrm{B}$ consensus site (5'-GGGACTTTCC-3'), attached to a 96-well plate. Assays were performed in duplicate, according to the manufacturer's instructions. NF- $\kappa \mathrm{B}$ DNA binding activity was determined as absorbance values measured by using an Anthos 2010 
ELISA reader. Increase in fluorescence was linear over extract concentration.

\section{Statistical analysis}

The media, median, minimum and maximum values were calculated for each group of data. For each set of experiments, values are reported as means \pm S.D. For selected experiments, results are reported as box plots showing the median, minimum and maximum values and 25 th to 75 th percentiles. Data were analysed by ANOVA and with the Mann-Whitney rank-sum test. Comparison of group means was performed by Bonferroni method. Statistical significance was defined as $P<0.05$.

\section{Results}

SC5b-9 and OPG are elevated in the plasma of patients affected by active $R A$ and co-localize in the endothelium of synovial tissue

In the first set of experiments, we measured the levels of SC5b-9 and OPG in the plasma and SF of patients with active RA. As shown in Fig. 1A, SC5b-9 and OPG plasma levels were significantly $(P<0.05)$ increased in RA patients compared with the healthy controls. Interestingly, however, while the levels of SC5b-9 were only slightly increased in the SF of RA patients with respect to the plasma of the same patients, OPG levels were further increased $(P<0.01)$ in SF with respect to the plasma of RA patients (Fig. 1B). Of note, TNF- $\alpha$ was also significantly $(P<0.05)$ higher in SF with respect to the plasma of RA patients (Fig. 1B)

Immunohistochemical analysis of serial sections of synovial membrane samples obtained from RA patients revealed the presence of SC5b-9 deposits and OPG expression in the endothelium of vessels traversing the synovium, with a clear co-localization of SC5b-9 deposition and OPG-reactivity in the endothelium of small venulae capillaries (Fig. 2).

\section{Clinically relevant concentrations of $S C 5 b-9$ and $O P G$ promote PMN adhesion to endothelial cells}

It is well known that neutrophilic infiltration is a key step in the onset and maintenance of inflammatory joint diseases [36]. Since the first event in the extravasation of leucocytes is represented by the firm adhesion to the endothelial layer, experiments were then designed to investigate whether SC5b-9 and OPG, used at the concentrations detected in the plasma and/or SF were able to promote PMN/endothelial cell interactions. HUVECs were grown to confluence and exposed to either SC5b-9 or OPG. As shown in Fig. 3, treatment with SC5b-9 as well as with recombinant OPG significantly increase the adhesion of PMNs to endothelial cells, suggesting that the concentrations of SC5b-9 and OPG found
A

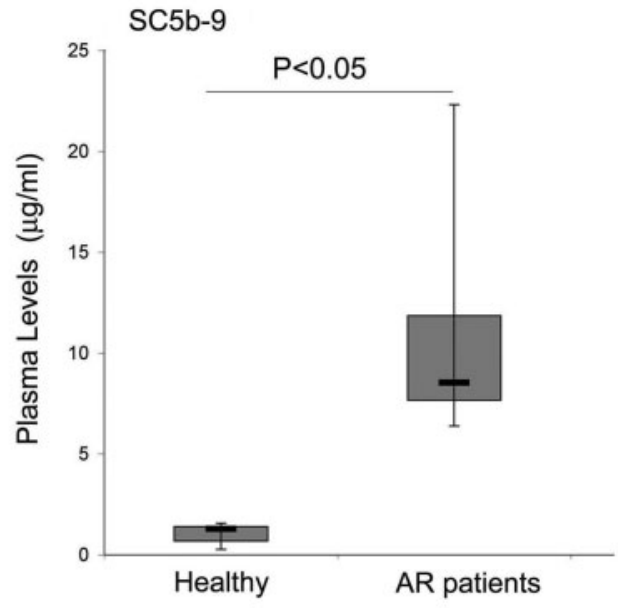

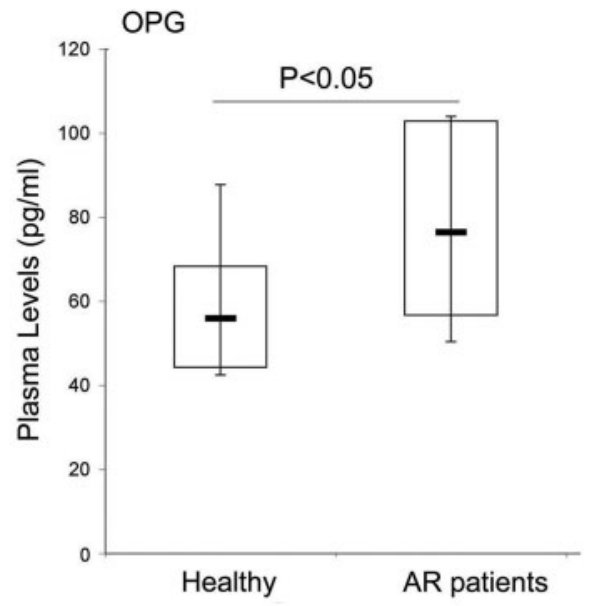
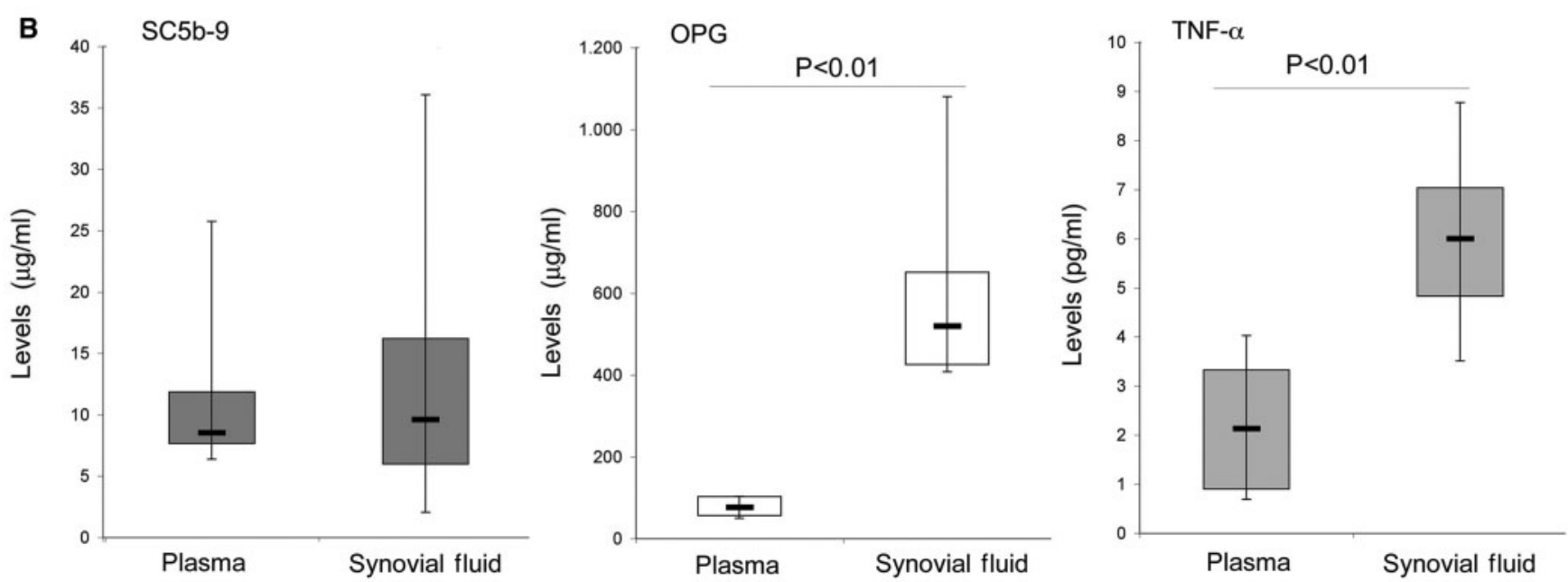

FIG. 1. High levels of SC5b-9 and OPG in RA patients. (A) Levels of SC5b-9 and OPG were determined by ELISA in plasma of RA patients and healthy control subjects.

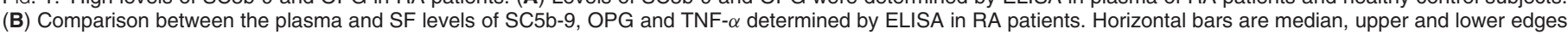
of box are 75th and 25th percentiles, lines extending from box are 10th and 90th percentiles. 
in plasma and in SF (Fig. 1A and B) may efficiently promote leucocyte extravasation in the synovia of RA patients.

\section{SC5b-9 up-regulates the expression and release of OPG by endothelial cells}

To investigate the potential link between SC5b-9 and OPG, we next examined the ability of SC5b-9 to modulate the release of OPG by endothelial cells. As expected [35], untreated HUVECs released low levels of OPG in the culture supernatants,

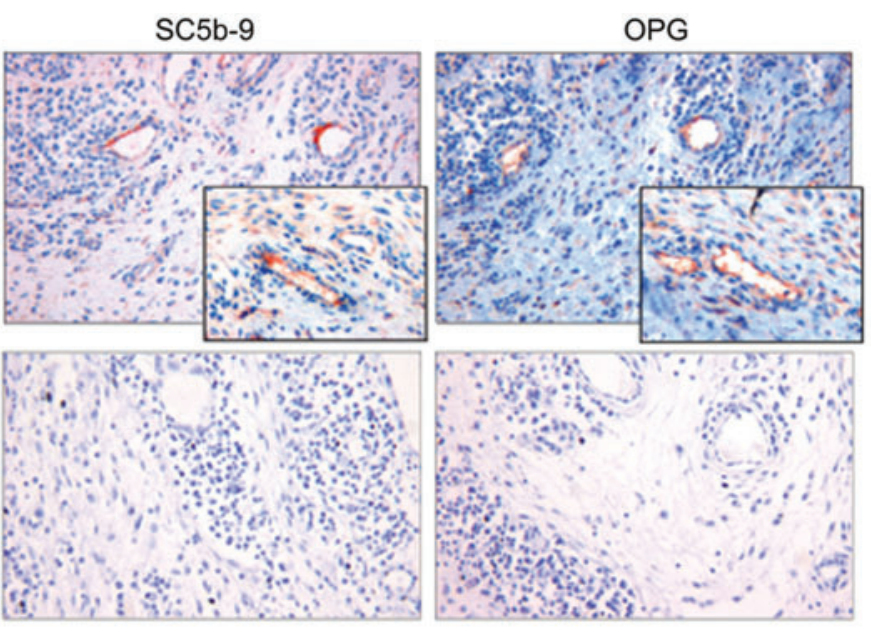

FIG. 2. SC5b-9 and OPG are co-localized on vascular endothelial cells in RA synovial membranes. Immunohistochemical evaluation of SC5b-9 and OPG staining on serial sections from synovial membrane samples. In the top panels, synovial membrane showed the presence of SC5b-9 deposition and intense OPG staining in the endothelium of vessels. In the bottom panels, negative controls of each immunohistochemical staining are shown. Original magnification, 200x; insets, $400 \times$. Representative results of five separate experiments that gave similar results are shown.

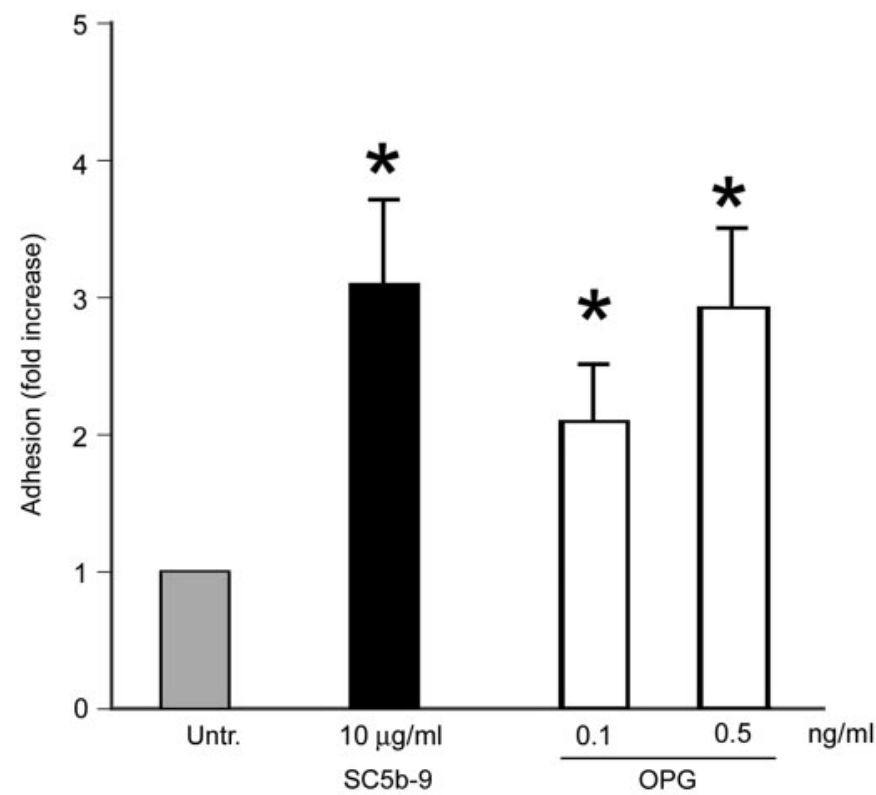

FIG. 3. Effect of SC5b-9 and OPG on PMN adhesion. HUVECs were either left untreated or exposed for $16 \mathrm{~h}$ to the indicated concentrations of SC5b-9 and OPG. After washing, PMNs were added to the endothelial monolayer, and the number of adhered cells was determined as described in Materials and methods section. Cell adherence on HUVECs is reported as fold of increase respect to cell adhesion in the absence of the indicated pre-treatment. Results are expressed as means \pm S.D. of five experiments, each performed in triplicate. ${ }^{*} P<0.05$. while exposure to SC5b-9, used at concentrations in the range of those detected in vivo, time- and dose-dependently up-regulated OPG release (Fig. 4A and B). Of note, the simultaneous treatment of HUVECs with SC5b-9 plus the pro-inflammatory cytokine TNF- $\alpha$, showed synergistic effects with respect to the production and release of OPG induced in response to SC5b-9 alone (Fig. 4C).

To ascertain whether SC5b-9 up-regulated OPG at the transcriptional level, we have generated a promoter-reporter construct containing the OPG promoter upstream of a luciferase reporter gene (pOPG-Luc). HUVECs were transfected with this construct, or with the empty control vector, and then were exposed to SC5b-9 for $18 \mathrm{~h}$ before assessing reporter gene activity. As shown in Fig. 5A, pOPG-Luc expression was significantly $(P<0.05)$ induced upon exposure of endothelial cells to SC5b-9. Taking into account that OPG is an NF- $\kappa \mathrm{B}$-dependent gene [37], we next evaluated the involvement of the $\mathrm{NF}-\kappa \mathrm{B}$ pathway in the up-regulation of OPG expression/release induced by SC5b-9.

A

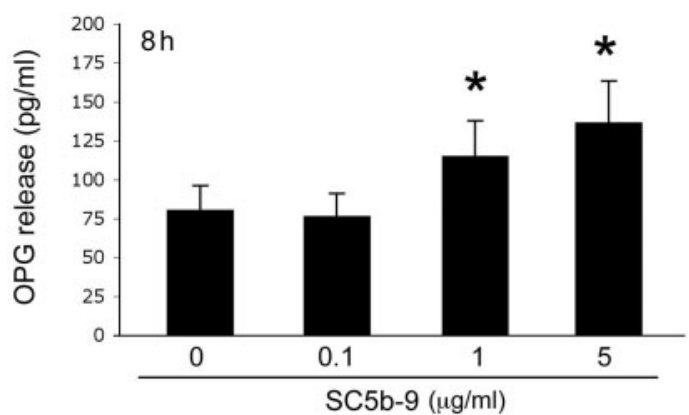

B
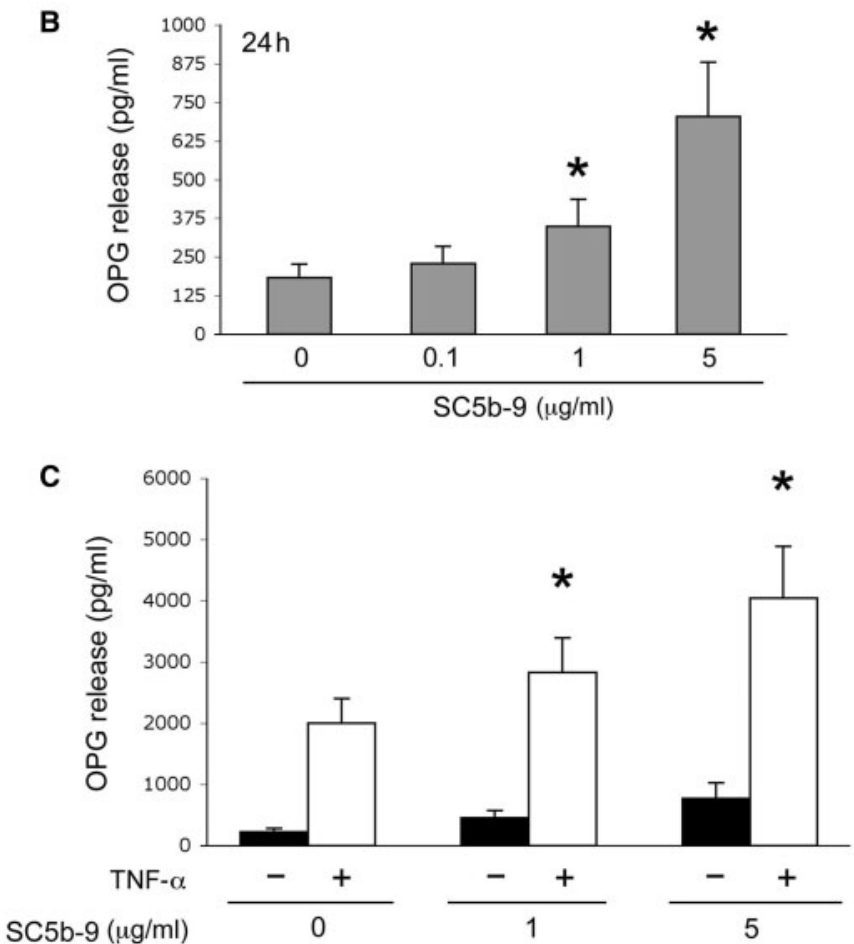

FIG. 4. SC5b-9 up-modulates OPG release in endothelial cells. HUVECs were left untreated or treated with the indicated concentrations of SC5b-9. OPG levels in culture supernatants were assessed by ELISA after $8 \mathrm{~h}(\mathbf{A})$ and $24 \mathrm{~h}(\mathbf{B})$ of treatment with SC5b-9 $(0.1-5 \mu \mathrm{g} / \mathrm{ml})$. ${ }^{*} P<0.05$, compared with untreated. (C) OPG levels in culture supernatants were assessed by ELISA after $24 \mathrm{~h}$ of treatment with the indicated concentrations of SC5b-9 used either alone (black bars) or in combination with TNF- $\alpha\left(1 \mathrm{ng} / \mathrm{ml}\right.$; white bars). ${ }^{*} P<0.05$, compared with SC5b-9. (A-C) Data are expressed as means \pm S.D. of results from four independent experiments, each performed in duplicate. 

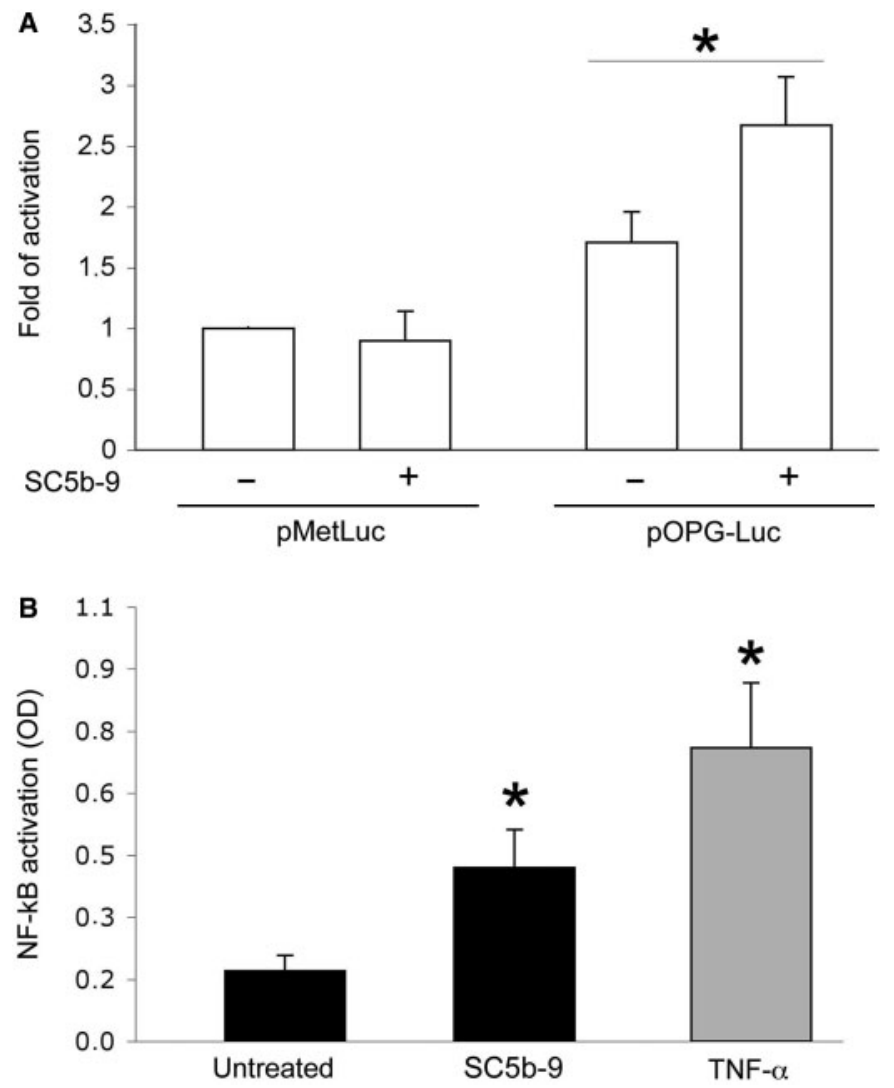

FIG. 5. SC5b-9 up-regulates OPG-promoter through activation of NF- $\kappa$ B. (A) HUVECs were transfected with pOPG-Luc or empty pMetLuc-Reporter vector. After recovery, transfected cells were stimulated as indicated and luciferase activity was assessed. pMetLuc was set as unit and results were calculated as fold of activation. Data are expressed as means \pm S.D. of three independent experiments. ${ }^{*} P<0.05$. (B) HUVECs were either left untreated or stimulated with SC5b-9 $(5 \mu \mathrm{g} / \mathrm{ml})$ for $30 \mathrm{~min}$. NF- $\kappa$ B-p65 DNA binding activity was determined as absorbance values (OD) per microgram of cell lysate protein. NF- $\kappa$ B activation by TNF- $\alpha(10 \mathrm{ng} / \mathrm{ml})$ is also shown for comparison. Results are expressed as means \pm S.D. of three independent experiments, each performed in duplicate. ${ }^{*} P<0.05$, compared with untreated cultures.

By using an oligonucleotide containing the $k \mathrm{~B}$ consensus site (TransAM), a significant induction of p65/RelA NF- $\kappa$ B-DNA binding over untreated cultures was observed after $30 \mathrm{~min}$ of SC5b-9-treatment (Fig. 5B).

\section{Discussion}

Although the data concerning the levels of OPG in the sera of patients affected by RA are heterogeneous [20-26], a couple of studies have clearly shown that OPG concentrations were higher in patients with long-standing RA with respect to early RA, which in turn showed OPG levels more elevated than in controls [21, 26]. Consistently, we have observed significantly higher levels of OPG in plasma of active RA patients with respect to healthy controls. The same RA patients were also characterized by a significant increase of the plasmatic soluble terminal complement complex SC5b-9, compared with the healthy controls. Starting from these observations, we have demonstrated, by a series of in vitro experiments, that concentrations of SC5b-9 compatible with those measured in the plasma and/or SF of RA patients, significantly up-regulated the release of OPG in endothelial cultures. Moreover, we have demonstrated that SC5b-9 induced OPG expression in endothelial cells acting at the transcriptional level, likely through the NF- $\kappa \mathrm{B}$ pathway. Although it has been shown that $\mathrm{OPG}$ is an NF- $\kappa \mathrm{B}$-dependent gene [37], and we have previously demonstrated that its expression and release in endothelial cells is induced by the pro-inflammatory cytokine TNF- $\alpha$ [35], this is the first study demonstrating that also the complement cascade, and in particular SC5b-9, significantly induce OPG expression and release by endothelial cells. Furthermore, we have observed that TNF- $\alpha$ synergized with SC5b-9 in further promoting OPG release by endothelial cells, suggesting that different inflammatory stimuli cooperate in promoting the expression and release of OPG by endothelial cells. In this respect, it is noteworthy that while the concentrations of SC5b-9 were only slightly higher in SF than in plasma of RA patients, OPG concentrations were significantly $(P<0.01)$ greater in SF with respect to the plasma of the same RA patients. Of note, TNF- $\alpha$ was also significantly $(P<0.05)$ higher in $\mathrm{SF}$ with respect to the plasma of RA patients. Thus, it is possible to suppose a cooperation between SC 5b-9 and TNF- $\alpha$ in SF, which results in the induction of the higher levels of OPG detected in SF vs plasma of RA patients. The relevance of our in vitro data was underscored by in situ immunohistochemical analysis of synovial lesions, which clearly showed a co-localization of SC5b-9 deposition and strong OPG expression in the endothelium, suggesting a potential link between these two molecules also in vivo. Moreover, the ability of both SC5b-9 and OPG to promote neutrophil-endothelial cell adhesion indicates that both molecules are actively involved in the tissue inflammation [36].

In conclusion, our data clearly indicate that OPG is a target gene of the complement-cascade and might contribute to the pro-inflammatory cascade characteristic of RA. Although different studies have originally focused the attention on beneficial effects of elevated OPG levels in counteracting the RANKLmediated induction of osteoclastogenesis and thus preventing bone erosion in RA patients [22-25], our data point to the possibility that elevated OPG levels both in the plasma and in the SF might rather contribute to pathogenetic aspects of RA, such as increased adhesiveness of PMNs to endothelial cells. Moreover, beside RANKL, OPG is able to interact with a comparable affinity with TRAIL, and accumulating data indicate that the interplay among RANKL, OPG and TRAIL is more complex than originally thought [38-42]. In this respect, it is noteworthy that cultured rheumatoid fibroblast-like synovial cells are susceptible to TRAIL-mediated apoptosis, but recombinant OPG inhibits the pro-apoptotic activity of TRAIL [43]. Thus, it has been proposed that inflammatory cytokines might promote the growth of rheumatoid synovial tissues indirectly, through stimulation of OPG production and its interference with TRAIL-death signal [43]. It should also be mentioned that the pro-adhesive and proinflammatory activities of OPG $[27,28]$ might be totally unrelated to its ability to neutralize the activity of RANKL and TRAIL.

\section{Rheumatology key messages}

- SC5b-9 induces OPG expression and release by endothelial cells.

- SC5b-9-mediated up-regulation of OPG may be an important mechanism whereby complement contributes in promoting and/or enhancing the inflammation in RA.

Funding: This work was supported by CariFe foundation and Ministero dell'Istruzione, Universitá e Ricerca (MIUR; PRIN 2007 grant).

Disclosure statement: The authors have declared no conflicts of interest.

\section{References}

1 Morgan BP. The complement system: an overview. Methods Mol Biol 2000; 150:1-13.

2 Neumann E, Barnum SR, Tarner IH et al. Local production of complement proteins in rheumatoid arthritis synovium. Arthritis Rheum 2002;46:934-45.

3 Hietala MA, Nandakumar KS, Persson L, Fahlén S, Holmdahl R, Pekna M. Complement activation by both classical and alternative pathways is critical for the effector phase of arthritis. Eur J Immunol 2004;34:1208-16. 
4 Petersen NE, Elmgreen J, Teisner B, Svehag SE. Activation of classical pathway complement in chronic inflammation. Elevated levels of circulating c3d and c4d split products in rheumatoid arthritis and Crohn's disease. Acta Med Scand 1988;223: 557-60.

5 Molenaar ET, Voskuyl AE, Familian A, van Mierlo GJ, Dijkmans BA, Hack CE. Complement activation in patients with rheumatoid arthritis mediated in part by C-reactive protein. Arthritis Rheum 2001:44:997-1002.

6 Wouters D, Voskuyl AE, Molenaar ET, Dijkmans BA, Hack CE. Evaluation of classical complement pathway activation in rheumatoid arthritis: measurement of C1q-C4 complexes as novel activation products. Arthritis Rheum 2006:54:1143-50.

7 Swaak AJ, Van Rooyen A, Planten O, Han H, Hattink O, Hack E. An analysis of the levels of complement components in the synovial fluid in rheumatic diseases. Clin Rheumatol 1987:6:350-7.

8 Brodeur JP, Ruddy S, Schwartz LB, Moxley G. Synovial fluid levels of complement SC5b-9 and fragment $\mathrm{Bb}$ are elevated in patients with rheumatoid arthritis. Arthritis Rheum 1991;34:1531-7.

9 Corvetta A, Pomponio G, Rinaldi N, Luchetti MM, Di Loreto C, Stramazzotti D. Terminal complement complex in synovial tissue from patients affected by rheumatoid arthritis, osteoarthritis and acute joint trauma. Clin Exp Rheumatol 1992;10:433-8.

10 Hogasen K, Mollnes TE, Harboe M, Gotze O, Hammer HB, Oppermann M. Terminal complement pathway activation and low lysis inhibitors in rheumatoid arthritis synovial fluid. J Rheumatol 1995;22:24-8.

11 Linton SM, Morgan BP. Complement activation and inhibition in experimental models of arthritis. Mol Immunol 1999;36:905-14.

12 Andersson SE, Lexmuller K, Ekstrom GM. Physiological characterization of mBSA antigen induced arthritis in the rat. I. Vascular leakiness and pannus growth. J Rheumatol 1998;25:1772-7.

13 Fischetti F, Durigutto P, Macor P, Marzari R, Carretta R, Tedesco F. Selective therapeutic control of $\mathrm{C} 5 \mathrm{a}$ and the terminal complement complex by anti-C5 singlechain Fv in an experimental model of antigen-induced arthritis in rats. Arthritis Rheum 2007;56:1187-97.

14 Tedesco F, Pausa M, Nardon E et al. The cytolytically inactive terminal complement complex activates endothelial cells to express adhesion molecules and tissue factor procoagulant activity. J Exp Med 1997;185:1619-27.

15 Bossi F, Fischetti F, Pellis $V$ et al. Platelet-activating factor and kinin-dependent vascular leakage as a novel functional activity of the soluble terminal complement complex. J Immunol 2004;173:6921-7.

16 Simonet WS, Lacey DL, Dunstan CR et al. Osteoprotegerin: a novel secreted protein involved in the regulation of bone density. Cell 1997;89:309-19.

17 Boyle WJ, Simonet WS, Lacely DL. Osteoclast differentiation and activation. Nature 2003;423:337-42.

18 Zauli G, Secchiero P. The role of the TRAIL/TRAIL-receptors system in hematopoiesis and endothelial cell biology. Cytok Growth Fact Rev 2006;17:245-57.

19 Zauli G, Rimondi E, Nicolin V, Melloni E, Celeghini C, Secchiero P. TNF-related apoptosis-inducing ligand (TRAIL) blocks osteoclastic differentiation induced by RANKL plus M-CSF. Blood 2004;104:2044-50.

20 Ziolkowska M, Kurowska M, Radzikowska A et al. High levels of osteoprotegerin and soluble receptor activator of nuclear factor kappa B ligand in serum of rheumatoid arthritis patients and their normalization after anti-tumor necrosis factor alpha treatment. Arthritis Rheum 2002;46:1744-53.

21 Asanuma Y, Chung CP, Oeser A et al. Serum osteoprotegerin is increased and independently associated with coronary-artery atherosclerosis in patients with rheumatoid arthritis. Atherosclerosis 2007:195:e135-41.

22 Vandooren B, Cantaert T, Noordenbos T, Tak PP, Baeten D. The abundant synovial expression of the RANK/RANKL/Osteoprotegerin system in peripheral spondylarthritis is partially disconnected from inflammation. Arthritis Rheum 2008:58:718-29.

23 Sennels HP, Sørensen S, Ostergaard M et al. Circulating levels of osteopontin, osteoprotegerin, total soluble receptor activator of nuclear factor-kappa B ligand, and high-sensitivity $\mathrm{C}$-reactive protein in patients with active rheumatoid arthritis randomized to etanercept alone or in combination with methotrexate. Scand $J$ Rheumatol 2008;37:241-7.

24 Haynes D, Crotti T, Weedon $\mathrm{H}$ et al. Modulation of RANKL and osteoprotegerin expression in synovial tissue from patients with rheumatoid arthritis in response to disease-modifying antirheumatic drug treatment and correlation with radiologic outcome. Arthritis Rheum 2008;59:911-20.

25 Hein GE, Meister M, Oelzner P, Franke S. SRANKL and OPG in serum and synovial fluid of patients with rheumatoid arthritis in comparison to non-destructive chronic arthritis. Rheumatol Int 2008;28:765-9.

26 Skoumal M, Haberhauer G, Kolarz G et al. The imbalance between osteoprotegerin and cathepsin $\mathrm{K}$ in the serum of patients with longstanding rheumatoid arthritis. Rheumatol Int 2008;28:637-41.

27 Zauli G, Corallini F, Bossi F et al. Osteoprotegerin increases leukocyte adhesion to endothelial cells both in vitro and in vivo. Blood 2007;110:536-43.

28 Mangan SH, Campenhout AV, Rush C, Golledge J. Osteoprotegerin upregulates endothelial cell adhesion molecule response to tumor necrosis factor-alpha associated with induction of angiopoietin-2. Cardiovascular Res 2007;76:494-505.

29 Arnett FC, Edworthy SM, Bloch DA et al. The American Rheumatism Association 1987 revised criteria for the classification of rheumatoid arthritis. Arthritis Rheum 1988;31:315-24.

30 Secchiero P, Corallini F, Pandolfi A et al. An increased osteoprotegerin (OPG) serum release characterizes the early onset of diabetes mellitus and may contribute to endothelial cell dysfunction. Am J Pathol 2006;169:2236-44.

31 Zauli G, Pandolfi A, Gonelli A et al. TNF-related apoptosis-inducing ligand (TRAIL) sequentially up-regulates nitric oxide and prostanoid production in primary human endothelial cells. Circ Res 2003;92:732-40.

32 Dobrina A, Pausa M, Fischetti F et al. Cytolytically inactive terminal complement complex causes transendothelial migration of polymorphonuclear leukocytes in vitro and in vivo. Blood 2002:99:185-92.

33 Milani D, Zauli G, Rimondi E et al. Tumour necrosis factor-related apoptosis-inducing ligand sequentially activates pro-survival and pro-apoptotic pathways in SK-N-MC neuronal cells. J Neurochem 2003;86:126-35.

34 Secchiero $\mathrm{P}$, Lamberti $\mathrm{G}$, Corallini $\mathrm{F}$ et al. Conjunctival sac fluid contains elevated levels of soluble TRAIL: implications for the anti-tumoral surveillance of the anterior surface of the eye. J Cell Physiol 2009;218:199-204.

35 Secchiero $\mathrm{P}$, Corallini $\mathrm{F}$, Rimondi $\mathrm{E}$ et al. Activation of the $\mathrm{p} 53$ pathway downregulates the osteoprotegerin (OPG) expression and release by vascular endothelial cells. Blood 2008;111:1287-94.

36 Auer J, Bläss M, Schulze-Koops $\mathrm{H}$ et al. Expression and regulation of CCL18 in synovial fluid neutrophils of patients with rheumatoid arthritis. Arthritis Res Ther 2007;9:R94.

37 Malyankar U, Scatena M, Suchland K, Yun T, Clark E, Giachelli C. Osteoprotegerin is an avb3-induced, NF- $\kappa$ B-dependent survival factor for endothelial cells. J Biol Chem 2000;275:20959-62.

38 Vitovski S, Phillips JS, Sayers J, Croucher PI. Investigating the interaction between osteoprotegerin and RANKL or TRAIL: evidence for a pivotal role for osteoprotegerin in regulating two distinct pathways. J Cell Biol 2007;282:31601-9.

39 Brunetti G, Oranger A, Mori G et al. TRAIL is involved in human osteoclast apoptosis. Ann N Y Acad Sci 2007;1116:316-22.

40 Zauli G, Rimondi E, Stea S et al. TRAIL inhibits osteoclastic differentiation by counteracting RANKL-dependent p27Kip1 accumulation in pre-osteoclast precursors. J Cell Physiol 2008;214:117-25.

41 Zauli G, Rimondi E, Secchiero P. Soluble TRAIL does not impair the anti-osteoclastic activity of osteoprotegerin. J Cell Mol Med 2008;12:1063-5.

42 Chamoux E, Houde N, L'Eriger K, Roux S. Osteoprotegerin decreases human osteoclast apoptosis by inhibiting the TRAIL pathway. J Cell Physiol 2008;216: 536-42.

43 Miyashita T, Kawakami A, Nakashima T et al. Osteoprotegerin (OPG) acts as an endogenous decoy receptor in tumour necrosis factor-related apoptosis-inducing ligand (TRAIL)-mediated apoptosis of fibroblast-like synovial cells. Clin Exp Immunol 2004;137:430-6. 